\title{
Modern technology to support carers of care recipients with dementia or functional mental illness: promising progress, but a long road ahead
}

There is no doubt that family carers who look after a family member with dementia or with a functional mental illness fulfill an important role, not only for their loved one, but also for the health and aged care systems of the countries they live in. Due to increasing life expectancy, but also improved healthcare the number of family carers supporting older care recipients with functional mental illness or dementia is on the rise. While the carer role often can offer rewarding experiences caregivers are at increased risk of stress, depression, sleep problems, and often experience poor health outcomes with increased morbidity and mortality (Oyebode, 2003). Next to the stressors directly associated with the carer role, they often do not have the time to engage in healthy behavior to protect their physical, mental, and cognitive health (Loi et al., 2014). There is a wealth of literature providing evidence about effective strategies to support carers and the recent Lancet Commission on Dementia prevention, intervention, and care highlighted the importance of exploring how the use of technological innovations could support carers better (Livingston et al., 2017). The use of modern technology in this context can mean a variety of approaches, such as internet-based programs to provide education and skill-building, virtual support to assist with monitoring and managing challenging behavior, online support groups, and the use of assistive or therapeutic technology to improve safety, enable positive activities, and support communication between carer and care recipient, to name just a few (D'Onofrio et al., 2017; Ienca et al., 2017; Livingston et al., 2017). More specifically, telehealth approaches via videoconferences have the potential to better support carers who live in rural or remote regions (O'Connell et al., 2014) or who cannot attend faceto-face support programs for other reasons such as inability to leave the care recipient alone at home, being a multiple carer or having a disability themselves to give just some examples.

We recently conducted an international pilot study with an internet-based information and skillbuilding program (called RHAPSODY) specifically developed for family carers of care recipients with young onset dementia (YOD) (Kurz et al., 2016). Family carers of care recipients with YOD have specific needs due to common difficulties diagnosing the cause of dementia accurately and promptly and due to significant carer burden (Millenaar et al., 2016) and therefore require YOD specific information programs. RHAPSODY was well received by the family carers (unpublished data), but feedback from carers included the wish to be able to discuss what was learned via RHAPSODY in the context of their individual carer situation. We therefore will trial as a next step in a small pilot study an offer for carers to participate in two additional individual support sessions provided via communication technology after they have explored and used RHAPSODY.

Next to family carers, another group of carers who might receive additional support from modern technology for their work are professional carers working in the community, in hospitals, or residential care settings. We recently conducted a few small pilot studies to investigate some modern technology related benefits or barriers in "real life" clinical scenarios. For example, in one small pilot study, we were interested to find out whether older residents with functional mental illness (e.g. bipolar disorder, depression, anxiety, and schizophrenia) living in a low-level aged care residential facility would be able to engage in touch screen technology (TT) and whether this might help to improve social isolation and self-esteem (Loi et al., 2017a; 2017b). Professional carers involved in this study were occupational therapists and nurses who worked in these facilities and knew the residents well. The professional carers facilitated for six weeks, twice weekly 45-minute sessions to help the residents to learn to use TT with focusing on chosen topics of interest. While qualitative feedback from the residents was positive, social isolation and self-esteem did not improve significantly. Furthermore, the professional staff identified barriers such as participants experiencing fluctuating levels of concentration and attention which could lead to frustration when trying to 
learn and practice the new skill of using TT, so in these cases, potentially making their carer role more challenging.

In another small pilot study, we focused on training professional staff (registered nurses, enrolled nurses, allied health clinicians, personal care attendants, and domestic staff) who look after residents with dementia in three different residential aged care facilities in the Melbourne metropolitan area, in using TT (in this case in the form of Apple iPads ${ }^{\mathrm{TM}}$ or Android tablets) with their residents (Goh et al., 2017). TT had been available to be used by residents in these facilities prior to the study, but professional carers had indicated that they lacked confidence to introduce residents in using TT. One 40-minute educational session was offered to the mainly female and middle-aged staff with the aim to increase confidence and skills in using TT with the residents, e.g. how to use TT in general and how to use different applications of interest. After the session carers were significantly more confident in engaging residents with dementia in using TT, but there was no change in relation to their confidence in their own ability to use $\mathrm{TT}$, potentially indicating that TT training might need to be integrated for a longer period as part of overall training for professional carers. Another approach to support professional carers in residential care is to use modern technology to help them to use non-pharmacological strategies when managing challenging behaviors. In a recent pilot study, we demonstrated that the use of applications (apps) via TT by professional staff for a duration of three weeks to engage residents was effective in significantly reducing challenging behaviors in residents with dementia or schizophrenia compared to a control intervention and to usual care (Loi et al., 2017a; 2017b). However, staff also identified barriers for the use of apps, such as lack of confidence and lack of time. Focusing on family carers and technology to manage behavioral and psychological symptoms of dementia (BPSD), Kales et al. (2017) used focus groups to investigate carers' perceptions of what an app should include to support them to manage BPSD. Similarly to formal carers, minimizing training time, ease-touse technology and individual psychoeducation was reported to be important (Kales et al., 2017). The resulting WeCareAdvisor app is currently involved in a randomized controlled trial.

In a recent review on technology-based tools in the context of dementia care, several knowledge limitations and barriers were highlighted such as the limited evidence that these tools can reduce carer burden effectively, and the lack of studies focusing on institutional care compared to community care (Lorenz et al., 2017). In general, there is a lack of larger and randomized controlled studies. Furthermore, potential risks need to be better addressed, such as for the topic of data protection, ethical considerations, as well as the need to avoid misusing modern technology to replace social contact (Livingston et al., 2017; Lorenz et al., 2017).

From our experience, we conclude that more studies are needed to focus on carers of care recipients with functional mental illness and on professional carers working in hospitals and residential care. For family carers in the community, the combination of different types of modern technologies (e.g. information technology with communication technology) might offer more flexible approaches to tailor support for individual and changing needs. No matter what the care setting is, the use of modern technology needs to be easy to learn, time efficient and affordable to have a realistic chance to become a regular core component of high-quality care and carer support.

\section{Conflict of interest}

NT Lautenschlager was the Editor-in-Chief of International Psychogeriatrics at the time of submission and therefore this editorial was assigned to one of the Deputy Editors of International Psychogeriatrics for the editorial review and approval process.

Nicola T. LAUTENSChlager, ${ }^{1,2,3}$

JaNine Diehl-Schmid, ${ }^{4}$ Samantha M. LoI, ${ }^{1,2,5}$ JohanNes MAYer, ${ }^{4}$ MARIa Tensil ${ }^{4}$

AND Alexander F. KURZ

${ }^{1}$ Academic Unit for Psychiatry of Old Age, Department of Psychiatry, The University of Melbourne,

Melbourne, Australia

${ }^{2}$ NorthWestern Mental Health, Melbourne Health, Melbourne, Australia

${ }^{3}$ Institute for Advanced Study, Technische Universität

München, Munich, Germany

${ }^{4}$ Department of Psychiatry, Technische Universität

München, Munich, Germany

${ }^{5}$ Neuropsychiatry Unit, Royal Melbourne Hospital,

Parkville, Australia

Email: nicolatl@unimelb.edu.au

\section{Acknowledgments}

This work was supported by the Technische Universität München - Institute for Advanced Study, funded by the German Excellence Initiative. 


\section{References}

D'Onofrio, G. et al. (2017). Information and communication technologies for the activities of daily living in older patients with dementia: a systematic review. Fournal of Alzheimer's Disease, 57, 927-935.

Goh, A. M. Y., Loi, S. M., Westphal, A. and Lautenschlager, N. T. (2017). Person-centered care and management via technology of residents with dementia in aged care facilities. International Psychogeriatrics, doi: $10.1017 /$ S1041610217001375.

Ienca, M. et al. (2017). Intelligent assistive technology for Alzheimer's disease and other dementias: a systematic review. Fournal of Alzheimer's Disease, 56, 1301-1340.

Kales, H. C. et al. (2017). WeCareAdvisor. The development of a caregiver-focused, web-based program to assess and manage behavioural and psychological symptoms of dementia. Alzheimer Disease and Associated Disorders, 31, 263-270.

Kurz, A. et al. (2016). RHAPSODY - Internet-based support for caregivers of people with young onset dementia: program design and methods of a pilot study. International Psychogeriatrics, 28, 2091-2099.

Livingston, G. et al. (2017). Dementia prevention, intervention, and care. The Lancet, Available at: http://dx.doi.org/10.1016/S0140-6736(17)31363-6.

Loi, S. M. et al. (2014). Physical activity in caregivers: what are the psychological benefits? Archives of Gerontology and Geriatrics, 59, 204-210.
Loi, S. M., Hodson, S., Huppert, D., Swan, J., Mazur, A. and Lautenschlager, N. T. (2017a). Can a short internet training program improve social isolation and self-esteem in older adults with psychiatric conditions? (brief report). International Psychogeriatrics, 28, 1737-1740.

Loi, S. M., Mazur, A., Huppert, D., Hoy, B., Swan, J. and Lautenschlager, N. T. (2017b). A pilot study using "apps" as a novel strategy for the management of challenging behaviors seen in people living in residential care. International Psychogeriatrics, 29, 637-643.

Lorenz, K., Freddolino, P. P., Comas-Herrera, A., Knapp, M. and Damant, J. (2017). Technology-based tools and services for people with dementia and carers: mapping technology onto the dementia care pathway. Dementia, doi: 10.1177/1471301217691617.

Millenaar, J. K. et al. (2016). The care needs and experiences with the use of services of people with young-onset dementia and their caregivers: a systematic review. International fournal of Geriatric Psychiatry, 31, 1261-1276.

O'Connell, M. E. et al. (2014). Development and evaluation of a telehealth videoconferenced support group for rural spouses of individuals diagnosed with atypical early-onset dementias. Dementia, 13, 382-395.

Oyebode, J. (2003). Assessment of carers' psychological needs. Advances in Psychiatric Treatment, 9, 45-53. 